\title{
Triagem funcional dos participantes de um centro de referência ao idoso em Juazeiro do Norte
}

\author{
Jaciara B. Marques, ${ }^{1 *}$ Mariangela Perez, ${ }^{2,3}$ Roberto A. Lourenço ${ }^{2,3}$
}

\section{Resumo}

A condição funcional do idoso constitui-se um dos parâmetros mais importantes da avaliação geriátrica. A triagem funcional do idoso é um instrumento utilizado para identificar os principais problemas que podem levá-lo a alterações capazes de interferir no desempenho das atividades cotidianas. O presente estudo tem como objetivo apresentar o perfil de funcionalidade dos idosos participantes de um grupo de convivência do Centro de Referência ao Idoso de Juazeiro do Norte, município do Ceará. Foi aplicada a triagem funcional e questionário para identificação dos fatores sociodemográficos, autopercepção de saúde e aspectos da assistência à saúde, sendo avaliados 103 idosos. A maioria da amostra foi composta por mulheres, com idade acima de 70 anos, sem companheiro, em condição de coabitação, com baixa escolaridade e baixa renda. A maior parte dos avaliados classificou sua saúde como regular e ruim/muito ruim, relatou presença de doenças crônicas, mas negou hospitalização nos últimos doze meses. Dificuldade no ambiente domiciliar, suspeita de deficit cognitivo e dificuldade visual foram os indicadores de perda funcional mais detectados no estudo. Pequena parcela foi identificada com dependência nas atividades básicas e instrumentais de vida diária. Observou-se que todos os indicadores de perda funcional se apresentaram com aumento percentual no grupo de idade mais avançada e no grupo daqueles que tiveram autopercepção negativa da sua saúde. Através da triagem, foram detectados os indicadores de perdas funcionais, porém se faz necessária uma avaliação mais aprofundada dos idosos para melhor acompanhamento de sua saúde.

Descritores: Programas de rastreamento; Funcionalidade; Idoso.

\begin{abstract}
Functional screening of participants in a reference center for the elderly in Juazeiro do Norte in Ceará

The functional condition of the elderly constitutes one of the most important parameters of geriatric assessment. Functional screening of the elderly is a tool used to identify the problems can lead to functional changes that can affect the performance of activities of daily living. This article focuses on the describe of the functionality profile the individuals participating in the support group of the Reference Center on Elderly, Juazeiro do Norte, a municipality in the interior of Ceará and functional screening questionnaire was applied to identify the sociodemographic factors, self-rated health and aspects of health care, and 103 elderly was evaluated. The majority of the sample consisted of females, aged 70 years,
\end{abstract}

1. Departamento de Gerontologia. Faculdade de Medicina. Universidade Estácio de Sá. Juazeiro do Norte, CE, Brasil.

2. Disciplina de Geriatria. Escola Médica de Pós-Graduação. Pontifícia Universidade Católica do Rio de Janeiro. Rio de Janeiro, RJ, Brasil.

3. Disciplina de Geriatria. Departamento de Medicina Interna. Faculdade de Ciências Médicas. Universidade do Estado do Rio de Janeiro. Rio de Janeiro, RJ, Brasil.

"Endereço para correspondência:

Av. Padre Cícero, 3.921/ 902, São José

Juazeiro do Norte, CE, Brasil. CEP: 63024-015.

E-mail: geriatriacariri@gmail.com

Revista HUPE, Rio de Janeiro, 2015;14(2):17-22

doi: $10.12957 /$ rhupe.2015.18348

Recebido em 11/08/2015. Aprovado em 13/08/2015.

unmarried, on condition of cohabitation, with low education and low income. Most reviews rated their health as fair and poor / very poor, denied hospitalization in the last twelve months and reported presence of chronic diseases. Difficulty in the home environment, suspected cognitive impairment and visual impairment were more indicative of functional loss detected in the study. Small portion was identified with dependence in basic and instrumental activities of daily living. It was observed that all the indicators of functional loss presented with percentage increase in the older age group and in the group of those who had a negative perception of their health. Through functional screening indicators of functional losses were detected, but it is necessary to further evaluate the elderly to better monitoring of your health.

Keywords: Mass screening; Functioning; Aged.

\section{Resumen}

Tamizaje funcional de los ancianos del Centro de Referencia del Anciano de Juazeiro do Norte en Ceará

El estado funcional del anciano constituye uno de los parámetros más importantes en una evaluación geriátrica. El acompañamiento funcional del anciano es una herramienta utilizada para identificar los principales problemas que puedan llevar al anciano a alteraciones capaces de interferir en el desempeño de las actividades cotidianas. La presente investigación tiene por finalidad presentar el desempeño de funcionamiento de los ancianos participantes del Grupo de 
Convivencia del centro de referencia del Anciano de Juazeiro do Norte Municipio del interior del Ceará en Brasil.Fue aplicado el acompañamiento funcional y cuestionarios para identificar los factores sociodemográficos, de autopercepción de salud y aspectos asistenciales de salud. Siendo evaluados 103 ancianos. La mayoría de las muestras fue compuesta por mujeres con edad superior de 70 años sin compañero, en condición de convivencia con baja escolaridad y bajos recursos. La mayoría de los que fueron evaluados clasificó su salud como regular / mala /muy mala manifestaron la presencia de enfermedades crónicas, sin embargo negaron hospitalizaciones en los últimos 12 meses. Dificultad en el ambiente domiciliar ,sospecha de déficit cognitivo y deficiencia visual ,fueron los marcadores

\section{Introdução}

O envelhecimento populacional é, atualmente, um fenômeno observado na totalidade das sociedades afluentes, assim como na maioria dos países de renda média. ${ }^{1}$ No Brasil, o ritmo de crescimento populacional dos idosos é bastante consistente; segundo Pesquisa Nacional por Amostra de Domicílio (PNAD), em 2009, o país contava com cerca de 21 milhões de pessoas com 60 anos ou mais de idade, correspondendo a cerca de $11,3 \%$ do total da população. ${ }^{2}$

Segundo o Censo Populacional de $2010,{ }^{3}$ na cidade de Juazeiro do Norte no estado brasileiro do Ceará, existiam 249.939 habitantes, dos quais 26.392 eram idosos, correspondendo a 10,6\% da sua população. Em estudo realizado neste município, ${ }^{4}$ observou-se que os idosos procuravam os serviços de saúde mais próximos à sua residência e havia necessidade de profissionais com formação em geriatria para atenção especializada. Em outro estudo realizado nesta mesma cidade, ${ }^{5}$ concluiuse que para um melhor acompanhamento dos longevos pelos médicos da atenção primária seriam necessárias a capacitação profissional, melhorias do suporte assistencial e mudanças organizacionais das equipes da estratégia de saúde da família (ESF).

Esta mesma inadequação entre demandas e respostas do Estado e do setor privado tem sido observada em outras regiões do Brasil, de tal forma que um estudo qualitativo realizado na cidade de Santos, estado de São Paulo, ${ }^{6}$ em que foram entrevistados profissionais da saúde integrantes das equipes da ESF, as necessidades sociais e psicológicas da população não estavam sendo atendidas, a despeito de intervenções mais bem-planejadas. Da mesma forma, Nunes e colaboradores ${ }^{7}$ concluíram que $60,6 \%$ de uma amostra de 388 idosos necessitavam de ajuda nas atividades de perdida funcional más identificados en la investigación . Una pequeña parte fue identificada con dependencias en las atividades básicas e instrumentales de la vida cotidiana Se observó que todos los marcadores de pérdida funcional se presentan con un aumento del promedio en el porcentaje del grupo con edad más avanzada y en el grupo de aquellos que tuvieron autopercepción negativa de su salud. Atraves de este acompãnamiento fueron identificados los marcadores de perdidas funcionales. Entretanto se hace necesária una evaluación mucho más detallada de los ancianos para mejor acompañamiento de su salud.

Palabras clave: Tamizaje masivo; Funcionamiento; Anciano.

instrumentais de vida diária, e que equilíbrio e mobilidade prejudicados, depressão, deficit cognitivo e idade acima de 80 anos estavam associados à dependência em atividades de vida diária.

De um ponto de vista biológico, o envelhecimento determina alterações fisiológicas e patológicas que causam impacto no desempenho das atividades de vida diária. A condição funcional de uma pessoa com idade avançada é um dos parâmetros mais importantes da avaliação geriátrica. ${ }^{8}$

Os problemas de saúde na velhice costumam apresentar-se em mais de um órgão ou sistema orgânico e, frequentemente, envolvem aspectos de natureza social e mental. Por essa razão, a avaliação de saúde precisa ser abrangente e deve buscar, de maneira sistemática, alterações clínicas, funcionais, cognitivas, do humor, além da identificação da rede social de suporte?.

Para melhor adequação do atendimento aos idosos foram criados instrumentos que facilitam o rastreamento de problemas de saúde por meio de ferramentas padronizadas, que possibilitam identificar suas necessidades. Desde que treinado, qualquer profissional de saúde pode utilizar estes instrumentos. ${ }^{10}$

Entre estes instrumentos, os destinados a avaliação rápida de múltiplos sistemas devem ser uma opção, quando estamos diante do dilema de avaliar a funcionalidade de uma grande demanda por serviços de saúde. A triagem funcional proposta por Lachs e colaboradores, ${ }^{11}$ em 1990, foi criada com o objetivo de identificar as principais alterações funcionais, que com frequência interferem no desempenho das atividades de vida diária de indivíduos idosos. Este instrumento, embora desenhado inicialmente para suprir as necessidades de médicos clínicos norte-americanos, pode ser utilizado em unidades de saúde. É composto por 11 itens, organi- 
zados no formato de perguntas, aferições antropométricas e testes de desempenho, com a intenção de fazer uma avaliação inicial de maneira rápida e sistematizada das seguintes áreas: visão, audição, membros superiores e inferiores, continência urinária, nutrição, cognição e afeto, atividade de vida diária, ambiente domiciliar e suporte social.

O presente trabalho teve como objetivo apresentar o perfil funcional, estratificado por faixa etária e autoavaliação de saúde dos usuários do grupo de convivência do Centro de Referência ao Idoso (CRI) de uma cidade da Região Nordeste do Brasil.

\section{Materiais e métodos}

Trata-se de um estudo transversal e quantitativo realizado no CRI da cidade de Juazeiro do Norte, no estado do Ceará, Brasil. O CRI foi inaugurado em 2011 e atende aos pacientes por livre demanda, além dos encaminhados pela ESF do município. Em 2014, um total de 400 participantes frequentava o CRI para realizar atividades físicas, ocupacionais e culturais; também, recebiam acompanhamento de educadores físicos, assistentes sociais, consultas fisioterápicas, psicológicas, de enfermagem e médicas.

A amostra do presente estudo foi formada por 103 idosos avaliados no período de 01 a 30 de setembro de 2014. Os critérios de inclusão foram: ser cadastrado e participar das atividades do CRI e ter aceitado participar do estudo. O critério de exclusão foi não incapacidade de responder ao questionário.

O instrumento de pesquisa foi composto por questionário estruturado com variáveis sociodemográficas, de estado de saúde e pela triagem funcional de Lachs. ${ }^{11}$

Os dados sociodemográficos foram sexo, idade em estratos etários (60-69; 70-79; 80 e mais anos); estado civil (com companheiro e sem companheiro); escolaridade (analfabeto; até quatro anos de estudo; mais de quatro anos de estudo); renda familiar (menos que um salário; de um a dois salários e mais de três salários mínimos por habitante na residência) e arranjo familiar (mora sozinho ou mora acompanhado). O estado de saúde foi avaliado através do autorrelato de doenças crônicas, de internação nos últimos 12 meses e pela autoavaliação da saúde, através da pergunta "Como o(a) senhor(a) considera sua saúde?", com as opções de resposta: muito boa/ boa; regular e ruim/muito ruim. A triagem funcional foi aplicada por médica geriatra e duas colaboradoras do CRI previamente treinadas; foram considerados indicadores de perdas funcionais: ter dificuldade para enxergar avaliado pela pergunta "Tem dificuldade para ver televisão ou fazer outra atividade devido a problemas visuais?"; não responder à pergunta "Qual o seu nome?", sussurrada com a face do examinador fora da visão direta do paciente; ter incontinência urinária (se o idoso perdeu urina e molhou roupas íntimas sem querer em, pelo menos, seis dias separados no último ano); não se banhar, vestir ou fazer compras sozinho; não tocar a nuca com ambas as mãos ou ser incapaz de pegar uma caneta; não andar ou fazer percurso de levantar-se da cadeira e percorrer três metros em mais de 12 segundos; ter índice de massa corpórea inferior a $22 \mathrm{~kg} / \mathrm{m}^{2}$; não evocar uma ou mais das palavras "carro", "vaso", "bola", memorizadas três minutos antes; sentirse triste a maior parte do tempo; confirmar ter escadas, tapete solto ou pouca iluminação em seu domicílio; não ter familiar, amigo ou vizinho com o qual possa contar em caso de doença ou emergência.

Para a análise dos dados utilizou-se médias e desvios-padrão das variáveis contínuas, além de frequências absolutas e relativas das variáveis categóricas. Os dados relacionados às características funcionais foram estratificados por faixa etária e pela autoavaliação da saúde.

O projeto do estudo foi aprovado pela comissão de ética em pesquisa da Faculdade de Juazeiro do Norte, parecer número 799880.

\section{Resultados}

Na tabela 1 são apresentados os resultados descritivos referentes ao perfil sociodemográfico dos 103 idosos participantes do estudo. A maioria era do sexo feminino (76,7\%), com idade abaixo de 80 anos $(89,4 \%)$, sem companheiro $(68,9 \%)$ e em condição de coabitação (73\%). Destacaram-se as baixas escolaridade e renda dos participantes. A maioria dos avaliados classificou sua saúde como regular ou ruim/muito ruim, negou hospitalização nos últimos 12 meses e relatou presença de doenças crônicas.

O item de perda funcional mais frequente $(58,8 \%)$ foi dificuldade no ambiente domiciliar, como escadas, tapetes soltos e baixa iluminação, seguido pela suspeita de deficitcognitivo (57\%) e pela dificuldade visual (51\%).

A dependência nas atividades básicas e instrumentais de vida diária foi o indicador de perda funcional menos frequente $(5,9 \%)$, seguido por limitação de mobilidade dos membros superiores (7,9\%) e por ausência de suporte social (14,6\%).

Todos os indicadores de perda funcional aumen- 


\section{Artigo original}

Tabela 1. Perfil sociodemográfico e de estado de saúde dos participantes do Centro de Referência ao Idoso de Juazeiro do Norte, $n=103,2013$.

\begin{tabular}{l|c|c}
\hline \multicolumn{1}{c}{ Variável } & & $\mathrm{n}(\%)$ \\
\hline Sexo feminino & & $79(76,7)$ \\
\hline & $60-69$ & $36(35,0)$ \\
\hline Idade (anos) & $70-79$ & $56(54,4)$ \\
\hline Situação conjugal & $80+$ & $11(10,7)$ \\
\hline Arranjo familiar & Sem companheiro & $71(68,9)$ \\
\hline & Mora acompanhado & $75(73,0)$ \\
\hline Escolaridade (anos) & 0 & $49(49,0)$ \\
\hline Renda per capita & 1 a 4 & $38(38,0)$ \\
(salário mínimo) & $4+$ & $13(13,0)$ \\
\hline Autoavaliação de & Muito boa / Boa & $38(37,3)$ \\
saúde & Regular & $50(49,0)$ \\
\hline Hospitalização* (sim) & Ruim / Muito ruim & $14(13,7)$ \\
\hline Doença crônica (sim) & & $18(17,5)$ \\
\hline
\end{tabular}

\section{* Últimos 12 meses}

taram com a idade (Tabela 2) e com autopercepção negativa de sua saúde (Tabela 3).

\section{Discussão}

No presente estudo, a maioria dos entrevistados era do sexo feminino, com idade entre 65 e 79 anos, vivia sem companheiro, morava em condição de coabitação, tinha baixas escolaridade e renda, considerava sua saúde de regular a muito ruim e apresentava doenças crônicas. Quase metade da amostra (49\%) era analfabeta e 15 indivíduos $(14,6 \%)$ relatavam falta de suporte social; nesse subgrupo, a maioria era de faixa etária mais avançada (60\%) e avaliava negativamente a sua saúde (80\%). A proporção de idosos com algum grau de incapacidade funcional e de limitação da mobilidade foi pequena.

Destaca-se, entre os 103 idosos avaliados, a proporção de pior avaliação de saúde. A importância da autoavaliação de saúde como um indicador de bemestar individual e coletivo é conhecida na literatura. A relação entre nível socioeconômico e saúde subjetiva tem sido relatada de forma consistente com piores avaliações de saúde entre os menos escolarizados e de menor renda, características observadas na amostra estudada. ${ }^{12}$
Portanto, o perfil evidenciado nesse estudo foi semelhante ao de outros trabalhos com populações similares. Em Iguatu, município do interior do Ceará, em um estudo ${ }^{13}$ que avaliou participantes de grupos de convivência, a idade média dos entrevistados foi de 69,8 anos, sendo 90,8\% formado por mulheres, 40\% de viúvos e 53,8\% dos participantes avaliaram sua saúde como regular. Em um estudo realizado na cidade de Pelotas, Rio Grande do Sul, ${ }^{14}$ foi avaliada a prevalência de autoavaliação de saúde regular/ruim entre adolescentes, adultos e idosos e verificou-se que foi maior entre mulheres idosas, de maior faixa etária, menor nível econômico e com alguma doença crônica.

Os segmentos socialmente mais vulneráveis necessitam de estratégias de promoção de hábitos saudáveis de vida, buscando assim o alcance de bons resultados no seu acompanhamento. Nesse sentido, participantes de grupo de convivência tendem a apresentar melhor qualidade de vida quando comparados aos que não participavam de grupos de terceira idade, segundo estudo realizado em Itabira - Minas Gerais. ${ }^{15}$

Dados do presente estudo apontam para uma heterogeneidade funcional dos idosos e percebe-se a importância da aplicação do instrumento de triagem na identificação dos pacientes que necessitam de uma avaliação mais ampla. Segundo Freitas e Miranda, ${ }^{8}$ a avaliação geriátrica ampla (AGA), concebida por Marjory Warren, em 1936, consiste no diagnóstico multidimensional, com ênfase na funcionalidade.É por meio desta avaliação que se pode fazer um balanço entre as perdas e os recursos para a reabilitação. Entretanto, sabe-se que os que mais se beneficiam desse procedimento são os idosos fragilizados ou com algum risco de fragilização, justificando o uso de instrumentos de rastreio, tal como a triagem funcional, para busca intensiva de tais alterações em um grande número de indivíduos.

Lourenço e colaboradores ${ }^{16}$ propuseram um modelo no qual a triagem funcional fosse utilizada para identificar os idosos que necessitariam ser encaminhados para um nível de maior complexidade de atenção, no qual se submeteriam à AGA. Ou seja, em um ambiente com grande heterogeneidade de pacientes, um instrumento de alta sensibilidade na detecção de distúrbios nas diferentes dimensões da saúde seria útil para a organização de serviços prestados aos idosos.

No presente trabalho, a iniciativa de estabelecer um processo sistematizado de rastreio de idosos vulneráveis, para que possam receber o tipo de atenção mais adequada às suas necessidades, baseou-se em experiências semelhantes de serviços de geriatria de algumas 
Tabela 2. Indicadores de perdas funcionais em função da faixa etária dos participantes do Centro de Referência ao Idoso de Juazeiro do Norte, n=103, 2013.

\begin{tabular}{|l|c|c|c|}
\hline \multicolumn{1}{|c|}{ Indicadores funcionais } & $\begin{array}{c}60-69 \\
\mathrm{n}(\%)\end{array}$ & $\begin{array}{c}70+ \\
\mathrm{n}(\%)\end{array}$ & $\begin{array}{c}\text { Total } \\
\mathrm{n}(\%)\end{array}$ \\
\hline Dificuldade visual & $15(28,8)$ & $37(71,2)$ & $52(51,0)$ \\
\hline Dificuldade auditiva & $09(25,0)$ & $27(75,0)$ & $36(35,3)$ \\
\hline Incontinência urinária & $15(41,6)$ & $21(58,4)$ & $36(35,3)$ \\
\hline Dependência nas ABIVD* & $02(33,4)$ & $04(66,7)$ & $06(05,9)$ \\
\hline Limitação dos membros superiores & $03(37,5)$ & $05(62,5)$ & $08(07,9)$ \\
\hline Limitação dos membros inferiores & $06(25,0)$ & $18(75,0)$ & $24(24,7)$ \\
\hline Suspeita de déficit cognitivo & $18(31,6)$ & $39(68,4)$ & $57(55,9)$ \\
\hline Suspeita de depressão & $11(34,4)$ & $21(65,6)$ & $32(31,4)$ \\
\hline Ambiente domicilar inseguro & $23(38,3)$ & $37(61,7)$ & $60(58,8)$ \\
\hline Ausência de suporte social & $06(40,0)$ & $09(60,0)$ & $15(14,6)$ \\
\hline
\end{tabular}

*ABIVD: atividades básicas e instrumentais de vida diária

Tabela 3. Indicadores de perdas funcionais em função da autoavaliação da saúde dos participantes do Centro de Referência ao Idoso de Juazeiro do Norte, n=103, 2013.

\begin{tabular}{|c|c|c|c|}
\hline Indicadores funcionais & $\begin{array}{c}\text { Muito boa/Boa } \\
\text { n (\%) }\end{array}$ & $\begin{array}{l}\text { Regular/Ruim } \\
\text { Muito ruim } \\
\text { n (\%) }\end{array}$ & $\begin{array}{l}\text { Total } \\
\text { n (\%) }\end{array}$ \\
\hline Dificuldade visual & $14(26,9)$ & $38(73,1)$ & $52(51,0)$ \\
\hline Dificuldade auditiva & $13(37,1)$ & $22(62,9)$ & $36(35,3)$ \\
\hline Incontinência urinária & $11(30,6)$ & $25(69,6)$ & $36(35,3)$ \\
\hline Dependência nas ABIVD* & $02(33,3)$ & $04(66,7)$ & $06(05,9)$ \\
\hline Limitação dos membros superiores & $02(25,0)$ & $06(75,0)$ & $08(07,9)$ \\
\hline Limitação dos membros inferiores & $07(29,2)$ & $17(70,8)$ & $24(24,7)$ \\
\hline Suspeita de déficit cognitivo & $23(41,1)$ & $33(58,9)$ & $57(55,9)$ \\
\hline Suspeita de depressão & $07(22,6)$ & $24(77,4)$ & $32(31,4)$ \\
\hline Ambiente domicilar inseguro & $17(28,3)$ & $43(71,7)$ & $60(58,8)$ \\
\hline Ausência de suporte social & $03(20,0)$ & $12(80,0)$ & $15(14,6)$ \\
\hline
\end{tabular}

*ABIVD: atividades básicas e instrumentais de vida diária

universidades brasileiras, em que a hierarquização da demanda é determinada por meio do uso sistemático de instrumentos de rastreamento de fragilidade.

Como principais limitações desse estudo, destacamse o número reduzido de indivíduos estudados, ser uma descrição de perfil, portanto transversal, dos usuários de um grupo de convivência que não permite generalizações dos seus resultados, dado o provável viés de seleção e a ausência de uma AGA dos indivíduos que apresentaram perdas à triagem funcional, com o objetivo precípuo de validar os achados do rastreamento. Contudo, pode-se apontar como aspecto positivo, a descrição da experiência de se estabelecer o rastreamento de fragilidade de idosos, através do uso de um instrumento de rápida e fácil aplicação, em um ambiente de saúde pública, em um município do interior do Nordeste brasileiro.

Como perspectivas futuras, o acompanhamento 


\section{Artigo original}

dos idosos rastreados com a aplicação de alguma forma de avaliação geriátrica mais abrangente, com diagnósticos e respectivas intervenções estabelecidas, permitirá avaliar o impacto da estratégia implementada pelo CRI de Juazeiro do Norte.

\section{Conclusões}

No presente estudo, a maioria dos participantes do CRI foi composta pelo sexo feminino, com idade acima de 70 anos, sem companheiro e em condição de coabitação com baixa escolaridade e baixa renda. A maior parte dos avaliados classificou sua saúde como regular e ruim/muito ruim, negou hospitalização nos últimos 12 meses e relatou presença de doenças crônicas. Dificuldade no ambiente domiciliar, suspeita de deficit cognitivo e dificuldade visual foram os indicadores de perda funcional mais detectados no estudo. Observou-se que todos os indicadores de perda funcional se apresentaram com aumento percentual no grupo de idade mais avançada e no grupo daqueles que tiveram autopercepção negativa da sua saúde. Através da triagem funcional foram detectados os indicadores de perdas funcionais, mas, para alguns, faz-se necessário uma avaliação geriátrica ampla que se caracteriza por ser mais aprofundada e detalhada.

\section{Referências}

1. Jacob Filho W, Mônaco TA. Geriatria no século XXI. In: Lopes AC. Tratado de Clínica Médica. Rio de Janeiro: Roca LTDA; 2006.4248-4253.

2. IBGE. Síntese de Indicadores Sociais: uma análise das condições de vida da população brasileira. Rio de Janeiro, 2010.

3. IBGE. Censo Populacional 2010 [acesso em 2014 Out]. Disponível em: http://www.cidades.ibge.gov.br/.

4. Marques J, Rocha GAB, Granja HA, et al. O serviço de saúde pública em Juazeiro do Norte na visão dos idosos. In:
Nobrega-Therrien SM, Almeida MI de, Andrade JT. Formação diferenciada: a produção de um grupo de pesquisa. Fortaleza: Ed.UECE; 2009.12:235-250.

5. Marques JB, Villela WV. Concepção dos médicos da atenção primária de um município do interior do Ceará sobre saúde do idoso. Rev Saude em Debate. 2012;93(36): 234-42.

6. Costa MFBN, Ciosak SI. Atenção integral na saúde do idoso no Programa Saúde da Família: visão dos profissionais de saúde. Rev Esc Enferm. USP. 2010;44(2):437-444.

7. Nunes DP, Nakatani AYK, Silveira EA, et al. Capacidade funcional, condições socioeconômicas e de saúde de idosos atendidos por equipes de Saúde da Família de Goiânia, GO, Brasil. Cien Saude Colet. 2010; 15(6): 2887-2898.

8. Freitas EV, Miranda RD. Avaliação Geriátrica Ampla. In: Freitas, EV, et al Tratado de Geriatria e Gerontologia. Rio de Janeiro: Guanabara Koogan; 2011. 970-78.

9. Lourenço RA, Paradela EMP. Instrumentos de avaliação em Geriatria. In: Freitas EV, et al. Tratado de Geriatria e Gerontologia. Rio de Janeiro: Guanabara Koogan; 2011. p. 979.

10. Lino V. Rastreamento de Problemas de Saúde e Instrumentos usados na avaliação geriátrica e gerontológica. In: Freitas EV, et al. Tratado de Geriatria e Gerontologia. Rio de Janeiro: Guanabara Koogan; 2011.1001-13.

11. Lourenço RA, Perez M, Sanchez MAS. Triagem funcional do idoso. In: Freitas EV, et al. Tratado de Geriatria e Gerontologia. Rio de Janeiro: Guanabara Koogan; 2011. p.1710.

12. Borim FSA, Barros MBA, Neri AL. Autoavaliação da saúde em idosos: pesquisa de base populacional no Município de Campinas, São Paulo, Brasil. Cad Saúde Pública. Rio de Janeiro; 2012. 28(4):769-780.

13. Oliveira Silva H, Carvalho MJAD, Lima FEL, et al. Perfil epidemiológico de idosos frequentadores de grupos de convivência no município de Iguatu, Ceará. Rev Bras Geriatr Gerontol. Rio de Janeiro; 2011.14(1):123-133.

14. Reichert FF, Loch MR, Capilheira MF. Autopercepção de saúde em adolescentes, adultos e idosos. Cien Saude Colet. 2012;17(12):3353-3362,

15. Almeida EA, Madeira GD, Arantes PMM, et al. Comparação da qualidade de vida entre idosos que participam e idosos que não participam de grupos de convivência na cidade de Itabira-MG. Rev Bras Geriatr Gerontol. Rio de Janeiro; 2010.13(3):435-443.

16. Lourenço RA, Martins CSF, Sanchez MAS, et al. Assistência ambulatorial geriátrica: hierarquização da demanda. Rev Saude Publica. 2005;39:311-8. 